\title{
Editorial: Novel Strategies in Drug Development Against Multifactorial Diseases
}

\author{
Cinzia Esposito ${ }^{1}$, Catrine Johansson ${ }^{2}$ and Simone Di Micco ${ }^{3 *}$ \\ ${ }^{1}$ Department of Molecular Life Sciences, University of Zurich, Zurich, Switzerland, ${ }^{2}$ Oxford NIHR BRU, Botnar Research Centre, \\ Oxford Centre for Translational Myeloma Research, Oxford University, Oxford, United Kingdom, ${ }^{3}$ European Biomedical Research \\ Institute of Salerno (EBRIS), Salerno, Italy
}

Keywords: multifactorial disease, drug discovery, drug resistance, biochemistry, drug repurposing

Editorial on the Research Topic

\section{Novel Strategies in Drug Development Against Multifactorial Diseases}

The complexity of multifactorial diseases, such as inflammation, cancer, neurodegenerative disorders, infectious pathologies represents a key obstacle in the success of therapeutic intervention. The occurrence of acquired drug resistance against molecular targeted therapies harnesses this biological complexity. In this context, multitargeting compounds can be a remarkable strategy for multifactorial disease treatment and to tackle drug resistance mechanisms. Indeed, in contrast to drugs that bind to a single target, the modulation of multiple macromolecules gives rise to additive and synergistic properties with the advantage of reduced side effects.

Despite the great potential of multitargeting drugs to treat multifactorial diseases, a limited number of these compounds have reached clinical trials or market. The selection of the right target combination and the design and identification of molecules endowed with a multi-bioactive profile,

\section{OPEN ACCESS}

Edited and reviewed by: Michael Kassiou,

The University of Sydney, Australia

*Correspondence:

Simone Di Micco s.dimicco@ebris.eu

Specialty section: This article was submitted to Medicinal and Pharmaceutical Chemistry, a section of the journal Frontiers in Chemistry

Received: 17 December 2021 Accepted: 04 January 2022 Published: 24 January 2022

Citation: Esposito C, Johansson C and Di Micco S (2022) Editorial: Novel Strategies in Drug Development Against Multifactorial Diseases.

Front. Chem. 10:838063. doi: 10.3389/fchem.2022.838063 particularly challenging for unrelated targets, plays a key role in successful drug development. Thus, novel computational, chemical, biological, and biophysical approaches are urgently required for achieving new effective therapeutic tools towards the treatment of multifactorial diseases. This Research Topic gathers three research studies and one review.

Di Micco et al. adopted a drug repurposing strategy (Giordano et al., 2018a) to rapidly attenuate the current COVID-19 pandemic, demonstrating that the zonulin octapeptide inhibitor AT1001 (Larazotide acetate), currently in phase III trials in celiac disease (Troisi et al., 2021), binds the $\mathrm{M}^{\text {pro }}$ catalytic domain through molecular modeling and fluorescence resonance energy transfer (FRET) assay investigation. These findings combine with the well-demonstrated effect of AT1001 in improving mucosal permeability in ALI/ARDS, proposing it as a specific anti-SARS-CoV-2 multitargeting therapy for the global epidemic. This work led to the first generation of AT1001 derivatives, showing interesting anti-SARS-CoV-2 activity (Di Micco et al., 2021).

Recently, the development of an in silico/synthesis pipeline has been reported to identify new inhibitors of the glutathione-dependent enzyme mPGES-1, a valuable macromolecular target in both cancer therapy and inflammation therapy, has been reported (Di Micco et al., 2018). Specifically, the proposed approach was based on the virtual screening of commercially available fragments featuring aryl halide moieties, which represent the basic partners for Suzuki-Miyaura reactions (Miyaura and Suzuki, 1995), a very suitable synthetic strategy leading to platforms highly prone to further chemical modifications (Giordano et al., 2018b). Di Micco et al. implemented their in silico strategy, introducing phenyl and phenylethyl substituents as molecular probes mimicking boronic acid to improve arylbromide selection. The authors also enriched the input aryl bromide libraries to increase the chemical diversity exploration of molecular scaffolds to develop potential clinical candidates. In particular, the computer-aided approach could be potentially applied to design membrane-associated proteins in 
eicosanoid and glutathione (MAPEG) metabolism superfamily inhibitors, to target the prostaglandin pathway at multiple macromolecular levels for a more effective and safer therapy.

Zhang et al. applied bioinfomatic approaches on selected datasets acquired from the Gene Expression Omnibus (GEO) database to identify novel functional pathways, and diagnostic/ prognostic biomarkers implicated in the pathogenesis of systemic juvenile idiopathic arthritis (sJIA), with as yet unmet medical need. The authors identified six hub genes and specifically suggested ARG1 and PGLYRP1 as potential biomarkers for the early diagnosis of sJIA. Furthermore, Zhang et al. revealed the contribution of the MAPK pathway and immune components such as platelets in the pathogenesis of sJIA paving the way for novel potential molecular targets for sJIA treatment.

Anthwal et al. surveyed the synthetic strategy to obtain 1, 3, 4 thiadiazole-based compounds endowed with different biological activities, such as anti-cancer, anti-viral, anti-diabetic properties. As the 1, 3, 4 thiadiazole could be considered a privileged structure (Di Micco et al., 2016), the authors suggested that it is suitable to develop anticonvulsant compounds.

The collected contributions provide different perspectives to develop multitarget compounds. This approach potentially represents an opportunity to obtain safer therapeutical treatment and to overcome drug resistance.

\section{REFERENCES}

Di Micco, S., Musella, S., Sala, M., Scala, M. C., Andrei, G., Snoeck, R., et al. (2021). Peptide Derivatives of the Zonulin Inhibitor Larazotide (AT1001) as Potential Anti SARS-CoV-2: Molecular Modelling, Synthesis and Bioactivity Evaluation. Ijms 22, 9427. doi:10.3390/ijms22179427

Di Micco, S., Spatafora, C., Cardullo, N., Riccio, R., Fischer, K., Pergola, C., et al. (2016). 2,3-Dihydrobenzofuran Privileged Structures as New Bioinspired lead Compounds for the Design of mPGES-1 Inhibitors. Bioorg. Med. Chem. 24, 820-826. doi:10.1016/j.bmc.2016.01.002

Di Micco, S., Terracciano, S., Cantone, V., Fischer, K., Koeberle, A., Foglia, A., et al. (2018). Discovery of New Potent Molecular Entities Able to Inhibit mPGES-1. Eur. J. Med. Chem. 143, 1419-1427. doi:10.1016/j.ejmech.2017.10.039

Giordano, A., Forte, G., Massimo, L., Riccio, R., Bifulco, G., and Di Micco, S. (2018a). Discovery of New erbB4 Inhibitors: Repositioning an Orphan Chemical Library by Inverse Virtual Screening. Eur. J. Med. Chem. 152, 253-263. doi:10.1016/j.ejmech.2018.04.018

Giordano, A., del Gaudio, F., Johansson, C., Riccio, R., Oppermann, U., and Di Micco, S. (2018b). Virtual Fragment Screening Identification of a Quinoline5,8-Dicarboxylic Acid Derivative as a Selective JMJD3 Inhibitor. ChemMedChem 13, 1160-1164. doi:10.1002/cmdc.201800198

\section{AUTHOR CONTRIBUTIONS}

All authors listed have made a substantial, direct, and intellectual contribution to the work and approved it for publication.

\section{FUNDING}

This research was supported in part by the projects: Fase 2, studio multicentrico aperto per determinare la sicurezza, tollerabilità ed efficacia della larazotide acetato per l'uso urgente in pazienti anziani a rischio per la prevenzione di danno acuto polmonare (ali) e la sindrome da distress respiratorio acuto (ards) associate a infezione da COVID19-CUP G58D20000240002-SURF 20004BP000000011; Fighting Cancer Resistance: Multidisciplinary Integrated Platform for a Technological Innovative Approach to Oncotherapies (Campania Oncotherapies).

\section{ACKNOWLEDGMENTS}

The authors thank all the contributors, reviewers, handling editors, and the editorial officials of Frontiers in Chemistry.

Troisi, J., Venutolo, G., Terracciano, C., Delli Carri, M., Di Micco, S., Landolfi, A., et al. (2021). The Therapeutic Use of the Zonulin Inhibitor AT-1001 (Larazotide) for a Variety of Acute and Chronic Inflammatory Diseases. Cmc 28, 5788-5807. doi:10.2174/09298667328666210104110053

Conflict of Interest: The authors declare that the research was conducted in the absence of any commercial or financial relationships that could be construed as a potential conflict of interest.

Publisher's Note: All claims expressed in this article are solely those of the authors and do not necessarily represent those of their affiliated organizations, or those of the publisher, the editors and the reviewers. Any product that may be evaluated in this article, or claim that may be made by its manufacturer, is not guaranteed or endorsed by the publisher.

Copyright (c) 2022 Esposito, Johansson and Di Micco. This is an open-access article distributed under the terms of the Creative Commons Attribution License (CC BY). The use, distribution or reproduction in other forums is permitted, provided the original author(s) and the copyright owner(s) are credited and that the original publication in this journal is cited, in accordance with accepted academic practice. No use, distribution or reproduction is permitted which does not comply with these terms. 